UUITP-12/02

hep-th/0210058

\title{
On the consistency of de Sitter vacua
}

\author{
Ulf H. Danielsson \\ Institutionen för Teoretisk Fysik, Box 803, SE-751 08 Uppsala, Sweden \\ ulf@teorfys.uu.se
}

\begin{abstract}
In this paper the consistency of the de Sitter invariant $\alpha$-vacua, which have been introduced as simple tools to study the effects of transplanckian physics, is investigated. In particular possible non renormalization problems are discussed, as well as non standard properties of Greens functions. We also discuss the non thermal properties of the $\alpha$-vacua and the necessity of $\alpha$ to change. The conclusion is that non of these problems necessarily exclude an application of the $\alpha$-vacua to inflation.
\end{abstract}

October 2002 


\section{Introduction}

The main driving force behind recent work on transplanckian physics in cosmology, is to find out whether physics beyond the Planck scale can give effects on the CMBR spectrum, [1-30]. A crucial ingredient in inflationary cosmology is that microscopic quantum fluctuations are magnified by inflation into macroscopic seeds for galaxy formation. In standard inflation the modes of the inflaton field can be carried back in time to eras when they were much smaller than the Planck scale. For small scales the expansion of the universe can be ignored and a unique vacuum can be chosen for the inflaton. This is the Bunch-Davies vacuum. But the construction ignores the Planck scale and the natural expectation that physics beyond the Planck scale is very different from physics at low energies, and not possible to describe using a quantum field theory. The hope would be that transplanckian physics modifies the standard scenario and that the effects are magnified through inflation to leave visible imprints in the CMBR fluctuations.

There are two main approaches to investigate whether the above proposal is viable. One possibility is to use specific models of transplanckian physics, examples include models involving non commutative geometry, and investigate how this will change the predictions of inflation. Another possibility is to leave the details of the transplanckian physics for later work, and impose a cutoff on the theory at the Planck scale, or some other scale like the strings scale, where fundamentally new physics is expected. Our ignorance of the high energy physics is then encoded in the choice of initial conditions for the field modes when they start out at planckian size. Contrary to the standard scenario, the initial conditions are imposed in a situation where the time dependence of the background can not be ignored. Various natural ways of choosing the vacuum, minimal uncertainty, adiabatic to all orders etc., now give different results. The Bunch-Davies vacuum remains as a possibility but there are also other possibilities that also can be argued to be natural. A conservative approach is then to investigate the span of possibilities and see what effects, if any, they give on the CMBR. In [27] it was argued that the generic size of the effects is expected to be of order $H / \Lambda$ in the spectrum compared with the Bunch-Davies vacuum. To claim smaller effects one would need specific information about the nature of transplanckian physics. It is important to note that the generic effect is not a simple change in normalization of the spectrum but has a definite signature in the form of a modulation.

The simplified approach discussed in [27] [29], which is not tied to specific models of transplanckian physics, allows for a detailed examination of the consequences and viability of the transplanckian proposal. As observed in 29 it essentially amounts to an investigation of the physics of the de Sitter invariant vacua introduced in [31] 32] [33] [34, some times called the $\alpha$-vacua. These vacua have also recently been discussed in the context of de Sitter holography, [35] 336].

The purpose of this note is to address recent discussions in the literature, in particular [37 [38] 39], where the consistency of the $\alpha$-vacua is questioned. In the first two of the above mentioned works it has been pointed out that quantum field 
theory with $\alpha$-vacua is not well understood. In particular there are problems with renormalizability and the definition of loop amplitudes, and there are also peculiarities in certain Greens functions that are claimed to suggest that the vacua does not make physical sense.

The outline of the note is as follows. In section two we review the construction of the $\alpha$-vacua, in section three we focus on the problems of non renormalizability, in section four we discuss the large scale structure of the Greens functions, in section five how $\alpha$ might change with time, in section six the non-thermal nature of the $\alpha$-vacua and, finally, we end with some conclusions.

\section{Review of $\alpha$-vacua}

Let us briefly review how the $\alpha$-vacua are constructed. We will focus on a single inflaton field, $\phi(x)$, and its quantization. The Wightman function $G^{+}\left(x, x^{\prime}\right)$ for the inflaton field is defined as

$$
G^{+}\left(x, x^{\prime}\right)=\left\langle\Omega\left|\widehat{\phi}(x) \widehat{\phi}\left(x^{\prime}\right)\right| \Omega\right\rangle=\int d^{3} k \phi_{\mathbf{k}}(x) \phi_{\mathbf{k}}^{*}\left(x^{\prime}\right),
$$

where the field is expanded in modes as

$$
\widehat{\phi}(x)=\int d^{3} k\left[\phi_{\mathbf{k}}(x) \widehat{a}_{\mathbf{k}}+\phi_{-\mathbf{k}}^{\dagger}(x) \widehat{a}_{\mathbf{k}}^{\dagger}\right] .
$$

Operators are equipped with hats in all expressions. We then assume that these modes are obtained through Bogolubov transformations from the Bunch-Davies modes according to

$$
\phi_{\mathbf{k}}(x)=A \phi_{\mathbf{k}, B D}(x)+B \phi_{-\mathbf{k}, B D}^{\dagger}(x)
$$

with

$$
|A|^{2}-|B|^{2}=1
$$

A convenient parametrization is to write

$$
A=\frac{1}{\sqrt{1-e^{\alpha+\alpha^{*}}}} \quad B=\frac{e^{\alpha}}{\sqrt{1-e^{\alpha+\alpha^{*}}}},
$$

where $\alpha=-\infty$ is the Bunch-Davies vacuum. For convenience we write

$$
\phi_{\mathbf{k}, B D}(x)=\phi_{k, B D}(\eta) e^{i \mathbf{k} \cdot \mathbf{x}},
$$

and demand, for simplicity, that

$$
\phi_{k, B D}(\eta)=\phi_{k, B D}^{\dagger}(-\eta)
$$

To proceed, let us recall a few elementary aspects of de Sitter geometry. The metric in terms of coordinates useful for inflationary cosmology is given by

$$
d s^{2}=d t^{2}-a(t)^{2} d \mathbf{x}^{2}
$$


where $a(t)=e^{H t}$ is the scale factor. In terms of the conformal time $\eta=-\frac{1}{a H}$ the metric becomes

$$
d s^{2}=\frac{1}{H^{2} \eta^{2}}\left(d \eta^{2}-d \mathbf{x}^{2}\right)
$$

The inflationary universe can be viewed as the upper right triangle of the full de Sitter space, which has a Penrose diagram with the shape of a square, where $\eta \rightarrow-\infty$ corresponds to the Big Bang, while $\eta \rightarrow 0$ is the infinite future. The lower half of de Sitter space can be covered if we also consider positive $\eta$. A crucial ingredient in the study of the $\alpha$-vacua is the antipodal map, which in the above coordinates takes $x=(\eta, \mathbf{x})$ into $\bar{x}=(-\eta, \mathbf{x})$, and acts like a map between the two halves of de Sitter space.

With the help of the antipodal map the Bogolubov transformation can now be written

$$
\phi_{\mathbf{k}}(x)=A \phi_{\mathbf{k}, B D}(x)+B \phi_{\mathbf{k}, B D}(\bar{x}),
$$

and the Wightman function becomes

$$
G^{+}\left(x, x^{\prime}\right)=|A|^{2} G_{B D}^{+}\left(x, x^{\prime}\right)+|B|^{2} G_{B D}^{+}\left(x^{\prime}, x\right)+A B^{*} G_{B D}^{+}\left(x, \bar{x}^{\prime}\right)+B A^{*} G_{B D}^{+}\left(\bar{x}, x^{\prime}\right) .
$$

After this brief review of the $\alpha$-vacua we will now turn to the main subject of the paper: do the $\alpha$-vacua make physical sense?

\section{$3 \quad$ Are loop amplitudes ill defined?}

In [37 38 problems with the definition of loop amplitudes were pointed out. In both papers one loop amplitudes were investigated and failed to give finite and well defined results. In [37] it was shown that non local counter terms in the action, involving insertions at image points, were needed. In 38 the analytic structure of the Greens functions indicated that pinched singularities in the loop integrations make the results ill defined. These problems are clearly of great interest and it is a challenge to make sense out of the field theory under these circumstances. But none of these problems are necessarily relevant to the issue of transplanckian physics in cosmology.

The reason is simple. The whole point with the transplanckian physics, as explained in the introduction, is to see whether effects beyond quantum field theory can be relevant for the detailed structure of the fluctuation spectrum of the CMBR. Without a planckian cutoff there is no reason to impose initial conditions at any finite scale. The only natural procedure is to go to the infinite past, when the modes are infinitely small, and make the choice there. For these small scales the expansion of the universe is irrelevant and there is a unique natural choice of vacuum. This would be the end of the story in a world without a Planck scale (and dynamical gravity) in which quantum field theory could be trusted to all energies. The issue of $\alpha$-vacua, and transplanckian physics, would never arise. In the real world we do expect quantum 
field theory to break down at high enough energy to be replaced by something else, presumably string theory. To find out what kind of effects this new physics might have, we can try to modify the transplanckian physics by hand. One way to gain control over the situation is to impose a cutoff and assume quantum field theory to hold for energies below the cutoff. It is now the issue of vacuum choice becomes important. Since the Planck scale is not infinitely smaller than the inflationary Hubble scale, the time dependence of the background has to be taken into account, and there is no unique natural vacuum. In fact, there are certain $\alpha$-vacua that are as natural a product of the unknown transplanckian physics as the Bunch-Davies vacuum. The modest proposal behind [27] is simply that we should allow for this uncertainty in the possible outcome and investigate the consequences.

To summarize, the issue of $\alpha$-vacua only appears when one takes a planckian cutoff into account. This is perfectly consistent with the results of [38. The $\alpha$-vacua not only needs a planckian cutoff to be of physical relevance, they need a planckian cutoff to make physical sense. In other words, the quantum field theory loop amplitudes needs planckian input to give well defined answers.

In this context one should note that a rough cutoff in the sum over momenta and energies in (四) at the Planck scale, regulates the standard singularities in the Greens functions that occur when the insertions can be joined by a light ray. But it is not really these singularities that make trouble, it is rather those that are due to the image charges. But, as is easily seen, all terms in the Wightman function in (11) are regulated with this procedure. With these regulated Greens functions finite loop amplitudes can be constructed, even though their values are sensitive to exactly how the cutoff is implemented, that is, the results depend on transplanckian physics. The main point is that in a quantum gravity theory, where the Planck scale plays an important role, space time points are expected to be effectively smeared to a size of order Planck scale. That is, it is meaningless to claim, for instance, that you sit exactly on a light cone.

Many of the problems with the $\alpha$-vacua are related to the image charges and their apparent non local nature. This is what we turn to next.

\section{Do image charges break causality?}

Another issue that has been brought up concerns the long distance behavior of the theory. As we have seen, the $\alpha$-vacua can easily be constructed by allowing for image charges on the wrong side of de Sitter space. Greens functions might therefore receive contributions directly from a source but also through the image. This has caused concerns that the theory does not make physical sense and one might worry about problems of, e.g., causality.

It is, however, important to bear in mind the physical interpretations of the various Greens functions. The ones which are important for causality are the commutator 
and the retarded Greens functions, which are independent of the choice of vacuum and always vanish outside of the light cone. When physics depending on these Greens functions is studied, there is no difference between the standard Bunch-Davies vacuum and the more general $\alpha$-vacua. Greens functions that do depend on the choice of vacua, and therefore are different for the $\alpha$-vacua, are, e.g., the Feynman propagator and the Hadamard function. These are the ones that exhibit the extra singularities, outside of the light cone, that have caused some worries. One should note, however, that these Greens functions in general are expected to be non zero outside the light cone; the Bunch-Davies vacuum is no exception. Contrary to the commutator and retarded Greens function they encode information about the vacuum and correlations in the vacuum fluctuations. Unorthodox behavior, like in the case of the $\alpha$-vacua, might seem surprising but does not necessarily imply that the theory is inconsistent.

Of particular interest in this context is the Hadamard function. Note that the Feynman propagator can be written in terms of the vacuum independent retarded and advanced Greens functions and the vacuum dependent Hadamard function. The extra singularities of the Hadamard function occur, if we consider points at equal time in Robertson-Walker coordinates, for points separated by the diameter of de Sitter space. They simply imply that the correlation between fluctuations at such points is enhanced. The vanishing of the commutator guarantees that this can not be used for communications and can not lead to any break of causality. The large separation between the points makes it a little difficult for an observer to actually measure the correlations, although it is suggested in 38 that interactions might facilitate this. As far as the transplanckian effects are concerned, this is, however, not really the main point. When inflation ends the full structure of the Hadamard function will become visible and available to the CMBR. As suggested in [29], the end of inflation transforms the meta observables of 41] into real observables. Unfortunately, the direct detection of correlations over distances of the order the de Sitter diameter is not possible. At the end of inflation, the modes relevant for the CMBR are much larger than the de Sitter diameter, and the direct as well as the image mediated fluctuations are hidden in the extremely small scales. The only aspect that is claimed to be of relevance for the CMBR is a possible large scale tail.

[39] reached the same conclusion as in the present work concerning the irrelevance of correlations over spatial distances in the Feynman propagator. The authors of [39] continued, however, by pointing out that even though the singularities are harmless, a propagator that behaves like in Minkowsky space for small distances does not suffer from them anyway. Small distances and Minkowsky behavior can certainly be reached in the present universe well above the Planck scale. But, as has already been emphasized, the situation is very different during inflation when the Planck scale might be just a few orders of magnitude smaller than the inflationary scale. In such a universe Minkowsky behavior can not be reached before transplanckian physics make quantum field theory irrelevant. This way of arguing for the Bunch-Davies vacuum therefore lacks force.

Finally, it should be noted that it is essential for the consistency of the vacua that 
we restrict ourselves to half of de Sitter space. As pointed out already in [32], the direction of time changes on the wrong side of de Sitter. This also guarantees that there is no retarded propagator connecting an image charge with anything on the right half of de Sitter space.円

\section{$5 \quad$ Can $\alpha$ change with $H$ ?}

In [39] it is argued that $\alpha$ can not vary with a changing $H$. The reason, according to [39], is that local physics can not know about how $H$ changes and consequently $\alpha$ must remain constant. Since present day physics requires $e^{\alpha}$ to be substantially lower than any value that would be of interest to inflation, and $\alpha$ can not change with time, all interesting effects due to transplanckian physics are excluded.

However, one should note that even the Bunch-Davies modes have a dependence on $H$. This is easily seen from the Fourier transform of a Bunch-Davies mode given by

$$
\phi_{\mathbf{k}, B D}(x)=-\eta H \frac{1}{\sqrt{2 k}} e^{-i k \eta}\left(1-\frac{i}{k \eta}\right) e^{i \mathbf{k} \cdot \mathbf{x}},
$$

where $k$ is the comoving momentum. The form when the mode is created at the fundamental scale is obtained by using $p=k / a=\Lambda$ and recalling that $\eta=-\frac{1}{a H}$. The result has a dependence on $H$, and the planckian physics preparing the modes in a Bunch-Davies state therefore needs to know about the value of $H$. In a pure quantum field theory world the dependence is naturally generated through the expansion of the universe while the mode is in the transplanckian regime. A further dependence on $H$ through $\alpha$ is not qualitatively much different and it is easy to see that such a dependence is quite natural and local. The scale factor depends on time through $a(t)=e^{H t}$, with the expansion of space as a local effect taking place everywhere. The conjugate time interval associated with a mode of energy $\Lambda$ is $1 / \Lambda$, and during this time interval space expands by a factor $e^{H(t+1 / \Lambda)-H t}=e^{H / \Lambda} \sim 1+\frac{H}{\Lambda}$. This is true also for spatial distances close to the Planck scale and it is therefore natural to expect effects of the order $\frac{H}{\Lambda}$. This is immediately obvious from looking at the Bunch-Davies modes above, but transplanckian physics might lead to an additional dependence on $\frac{H}{\Lambda}$ through $\alpha$.

The simplest, and most natural dependence, is that we have

$$
e^{\alpha} \sim \frac{H}{\Lambda}
$$

and that this remains true at all times. Not only during inflation. However, in [42] it has been pointed out that even this small value of $e^{\alpha}$ could be in conflict with present day measurements of high energy gamma rays. A vacuum of the type above, it is

\footnotetext{
${ }^{1}$ Note however recent work, [40], where other possibilities to handle this problem are discussed.
} 
claimed, would lead to a higher rate of particle creation than what is acceptable. The discrepancy is not large, considering the sensitivity of the argument to the detailed astro-physics involved - it is a matter of an order of magnitude or two - but it nevertheless suggests that a more complicated behavior than the one above might be needed. This is precisely the subject of [43] where the vacuum relaxes towards the Bunch-Davies vacuum once inflation is over. At any rate, high energy gamma rays could, as suggested in [42, be another way to probe planckian physics in an expanding universe. One should also investigate how these effects might vary due to local variations in $H$.

\section{$6 \quad$ Will the $\alpha$-vacua thermalize?}

As discussed in 35 38 39 the $\alpha$-vacua are not thermal. One effect of this is that a detector in an $\alpha$-vacuum will not end up in thermal equilibrium. An equilibrium will be reached [38] 39], but it will not be thermal in character and in general not obey the laws of detailed balance. For a detector with just two levels, equilibrium trivially implies detailed balance between the two levels even though the occupation numbers will be non standard. With three levels, however, the situation is more unusual. Instead of detailed balance between any two levels, there will be a net transition rate from, say, level one to level two, from level two to level three and then back to level one. This is a consequence of the non thermal nature of the background and does not imply any inconsistent physics of the detector. A question one can ask, however, is whether and how such a background can sustain itself. Will there be processes that act to thermalize the background?

In [39] it is argued based on holography and complementarity that this will indeed be the case. The main argument is that, according to a specific observer in de Sitter space, any perturbation will be redshifted as it approaches the de Sitter horizon and apparently boiled to pieces through the ever higher temperature that the perturbation experiences according to the observer. All perturbations will inevitably be thermalized. One would conclude from this that all traces of physics taking place on smaller scales than the horizon will be erased and the featureless and thermal BunchDavies modes are the only thing that remains. However, this is slightly problematic if we take a point of view based on the Robertson-Walker coordinates in (8). We can now follow the modes through the de Sitter horizon and see how they freeze when they expand and become larger than the de Sitter radius. The analogue would be to follow an observer who ventures inside a black hole and find that nothing peculiar or dramatic happens near the horizon. And this seems to be the appropriate point of view to take when discussing fluctuations generated through inflation. Holography and complementarity are intriguing concepts that could be important for cosmology, but for this particular exercise they do not seem to be relevant. As observers of the CMBR we are more in the position of an observer inside a black hole rather than one on the outside. 
A more conservative approach would be to predict the evolution of the fluctuations without thinking about holography. Any effect that would threaten to thermalize the $\alpha$-vacua must then be due to interactions. To be specific one could imagine a thermal background with a small perturbation that starts out at Planck scale. It is important to note that the temperature is very low with the thermal wave length of the order of the de Sitter radius itself. As a consequence a planckian mode is relatively safe in the beginning. As it redshifts, and its energy decreases towards the de Sitter temperature, the thermalization effects should become more important. This happens over a period of time a few times the inverse temperature. But at the same time the mode will expand past the horizon and freeze. One seems to conclude that the thermalization effects could be important in an interacting theory, but it is not clear that they will suppress any difference with respect to the Bunch-Davies vacuum by orders of magnitude. Much less wipe out any difference completely. Clearly more detailed work is needed to make a precise prediction.

\section{Conclusions}

In this short note we have discussed a few of the problems of transplanckian physics that can be addressed in the simplified framework of $\alpha$-vacua. Our conclusion is that the peculiar image singularities of the Feynman propagator, as well as problems with non renormalization, do not necessarily imply the ruling out of transplanckian effects. A true inconsistency at this level would have had dramatic implications. Without observational input we would have been able to exclude all models of transplanckian physics that result in other vacua than the Bunch-Davies, including the non commutative examples discussed in, e.g., [12]. We do not believe that such strong conclusions can be drawn from work done so far. We have furthermore considered the way $\alpha$ might change. We do not see any fundamental difficulties in this respect but note that the work of 42 shows that there are important constraints coming from present day physics. Issues of thermalization also need further investigation but we do not think that any argument based on holography put forward so far gives any meaningful constraints.

To summarize, we believe that the essence of the arguments of [39] is the following claim: it is not consistent to assume a fixed high energy cut off where unknown transplanckian physics delivers states different form the Bunch-Davies vacuum. An important point of the present paper is that possible problems are not visible in the cutoff theory. All problems must rely on claims about contributions from energy scales above the cutoff, energy densities etc., and speculations about transplanckian physics. Clearly, more work is needed to figure out how unorthodox transplanckian physics really can be. 


\section{Acknowledgments}

The author would like to thank Lars Bergström, Daniel Domert, Martin Olsson, Hector Rubinstein, Gary Shiu and Konstantin Zarembo for valuable discussions. The author is a Royal Swedish Academy of Sciences Research Fellow supported by a grant from the Knut and Alice Wallenberg Foundation. The work was also supported by the Swedish Research Council (VR).

\section{References}

[1] R. H. Brandenberger, "Inflationary cosmology: Progress and problems," arXiv:hep-ph/9910410.

[2] J. Martin and R. H. Brandenberger, "The trans-Planckian problem of inflationary cosmology," Phys. Rev. D 63, 123501 (2001) arXiv:hep-th/0005209.

[3] J. C. Niemeyer, "Inflation with a high frequency cutoff," Phys. Rev. D 63, 123502 (2001) arXiv:astro-ph/0005533.

[4] R. H. Brandenberger and J. Martin, "The robustness of inflation to changes in super-Planck-scale physics," Mod. Phys. Lett. A 16, 999 (2001) arXiv:astroph/0005432].

[5] A. Kempf, "Mode generating mechanism in inflation with cutoff," Phys. Rev. D 63, 083514 (2001) arXiv:astro-ph/0009209.

[6] C. S. Chu, B. R. Greene and G. Shiu, "Remarks on inflation and noncommutative geometry," Mod. Phys. Lett. A 16, 2231 (2001) arXiv:hep-th/0011241.

[7] J. Martin and R. H. Brandenberger, "A cosmological window on trans-Planckian physics," arXiv:astro-ph/0012031.

[8] L. Mersini, M. Bastero-Gil and P. Kanti, "Relic dark energy from trans-Planckian regime," Phys. Rev. D 64, 043508 (2001) arXiv:hep-ph/0101210.

[9] J. C. Niemeyer and R. Parentani, "Trans-Planckian dispersion and scaleinvariance of inflationary perturbations," Phys. Rev. D 64, 101301 (2001) arXiv:astro-ph/0101451.

[10] A. Kempf and J. C. Niemeyer, "Perturbation spectrum in inflation with cutoff," Phys. Rev. D 64, 103501 (2001) arXiv:astro-ph/0103225.

[11] A. A. Starobinsky, "Robustness of the inflationary perturbation spectrum to trans-Planckian physics," Pisma Zh. Eksp. Teor. Fiz. 73, 415 (2001) [JETP Lett. 73, 371 (2001)] arXiv:astro-ph/0104043. 
[12] R. Easther, B. R. Greene, W. H. Kinney and G. Shiu, "Inflation as a probe of short distance physics," Phys. Rev. D 64, 103502 (2001) arXiv:hep-th/0104102.

[13] M. Bastero-Gil and L. Mersini, "SN1A data and CMB of Modified Curvature at Short and Large Distances," Phys. Rev. D 65 (2002) 023502 arXiv:astroph/0107256].

[14] L. Hui and W. H. Kinney, "Short distance physics and the consistency relation for scalar and tensor fluctuations in the inflationary universe," arXiv:astroph/0109107.

[15] R. Easther, B. R. Greene, W. H. Kinney and G. Shiu, "Imprints of short distance physics on inflationary cosmology," arXiv:hep-th/0110226.

[16] M. Bastero-Gil, P. H. Frampton and L. Mersini, "Modified dispersion relations from closed strings in toroidal cosmology," arXiv:hep-th/0110167.

[17] R. H. Brandenberger, S. E. Joras and J. Martin, "Trans-Planckian physics and the spectrum of fluctuations in a bouncing universe," arXiv:hep-th/0112122.

[18] J. Martin and R. H. Brandenberger, "The Corley-Jacobson dispersion relation and trans-Planckian inflation," arXiv:hep-th/0201189.

[19] J. C. Niemeyer, "Cosmological consequences of short distance physics," arXiv:astro-ph/0201511.

[20] F. Lizzi, G. Mangano, G. Miele and M. Peloso, "Cosmological perturbations and short distance physics from noncommutative geometry," arXiv:hep-th/0203099.

[21] G. Shiu and I. Wasserman, "On the signature of short distance scale in the cosmic microwave background," arXiv:hep-th/0203113.

[22] R. Brandenberger and P. M. Ho, "Noncommutative spacetime, stringy spacetime uncertainty principle, and density fluctuations," arXiv:hep-th/0203119.

[23] S. Shankaranarayanan, "Is there an imprint of Planck scale physics on inflationary cosmology?," arXiv:gr-qc/0203060.

[24] N. Kaloper, M. Kleban, A. E. Lawrence and S. Shenker, "Signatures of short distance physics in the cosmic microwave background," arXiv:hep-th/0201158.

[25] R. H. Brandenberger and J. Martin, "On signatures of short distance physics in the cosmic microwave background," arXiv:hep-th/0202142.

[26] S. F. Hassan and M. S. Sloth, "Trans-Planckian effects in inflationary cosmology and the modified uncertainty principle," arXiv:hep-th/0204110.

[27] U. H. Danielsson, "A note on inflation and transplanckian physics," Phys. Rev. D 66, 023511 (2002) arXiv:hep-th/0203198. 
[28] R. Easther, B. R. Greene, W. H. Kinney and G. Shiu, "A generic estimate of trans-Planckian modifications to the primordial power spectrum in inflation," arXiv:hep-th/0204129.

[29] U. H. Danielsson, "Inflation, holography and the choice of vacuum in de Sitter space," JHEP 0207, 040 (2002) arXiv:hep-th/0205227.

[30] J. C. Niemeyer, R. Parentani and D. Campo, "Minimal modifications of the primordial power spectrum from an adiabatic short distance cutoff," arXiv:hepth/0206149.

[31] N. A. Chernikov and E. A. Tagirov, "Quantum theory of scalar field in de Sitter space-time," Ann. Inst. Henri Poincaré, vol. IX, nr 2, (1968) 109.

[32] E. Mottola, "Particle Creation In De Sitter Space," Phys. Rev. D 31 (1985) 754.

[33] B. Allen, "Vacuum States In De Sitter Space," Phys. Rev. D 32 (1985) 3136.

[34] R. Floreanini, C. T. Hill and R. Jackiw, "Functional Representation For The Isometries Of De Sitter Space," Annals Phys. 175 (1987) 345.

[35] R. Bousso, A. Maloney and A. Strominger, "Conformal vacua and entropy in de Sitter space," arXiv:hep-th/0112218.

[36] M. Spradlin and A. Volovich, "Vacuum states and the S-matrix in dS/CFT," arXiv:hep-th/0112223.

[37] T. Banks and L. Mannelli, "De Sitter vacua, renormalization and locality," arXiv:hep-th/0209113.

[38] M. B. Einhorn and F. Larsen, "Interacting Quantum Field Theory in de Sitter Vacua," arXiv:hep-th/0209159.

[39] N. Kaloper, M. Kleban, A. Lawrence, S. Shenker and L. Susskind, arXiv:hepth/0209231.

[40] M. K. Parikh, I. Savonije and E. Verlinde, "Elliptic de Sitter space: dS/Z(2)," arXiv:hep-th/0209120.

[41] E. Witten, "Quantum gravity in de Sitter space," arXiv:hep-th/0106109.

[42] A. A. Starobinsky and I. I. Tkachev, "Trans-Planckian particle creation in cosmology and ultra-high energy cosmic rays," arXiv:astro-ph/0207572.

[43] K. Goldstein and D. A. Lowe, arXiv:hep-th/0208167. 